University of Nebraska - Lincoln

DigitalCommons@University of Nebraska - Lincoln

July 1966

\title{
Study of Some Solutions of the One-Electron-Two-Center Problem for Heteronuclear Cases
}

\author{
L.Y. Wilson \\ University of Nebraska - Lincoln \\ Gordon A. Gallup \\ UNL,ggallup1@unl.edu
}

Follow this and additional works at: https://digitalcommons.unl.edu/physicsgallup

Part of the Physics Commons

Wilson, L.Y. and Gallup, Gordon A., "Study of Some Solutions of the One-Electron-Two-Center Problem for Heteronuclear Cases" (1966). Gordon Gallup Publications. 26.

https://digitalcommons.unl.edu/physicsgallup/26

This Article is brought to you for free and open access by the Research Papers in Physics and Astronomy at DigitalCommons@University of Nebraska - Lincoln. It has been accepted for inclusion in Gordon Gallup Publications by an authorized administrator of DigitalCommons@University of Nebraska - Lincoln. 
density over the experimental range studied here and for the experimental accuracy of the data presented herein.

\section{ACKNOWLEDGMENTS}

The authors wish to acknowledge the National Science Foundation for the over-all support of the study, the Ethyl Corporation for the supply of Ethyl Antioxidant-702, Tennessee Gas Transmission Company and Associated Oil and Gas Company for the generous supply of methane, the Phillips Petroleum Company for the research-grade propane, and Monsanto Chemical Company for gas-mixture analytical work.

\title{
Study of Some Solutions of the One-Electron-Two-Center Problem for Heteronuclear Cases*
}

\author{
L. Y. Wilson $\nmid$ and G. A. Galtup \\ Avery Laboratory, University of Nebraska, Lincoln, Nebraska
}

(Received 23 February 1966)

\begin{abstract}
The Schrödinger equation for two nuclei and one electron has been solved for the first eight $\sigma$ states of the system $\mathrm{HeH}^{2+}, \mathrm{He}_{2}{ }^{3+}, \mathrm{LiH}^{8+}$, and $\mathrm{LiHe}^{4+}$. The resulting solutions are described and some of their properties are discussed.
\end{abstract}

\section{INTRODUCTION}

$\mathbf{T}$ HE solutions of the hydrogenlike atom problem in quantum mechanics occupy a very important position in the theory of the structure of complex atoms. This is due in part to the relative simplicity of the functional forms in this case, of course. A similar situation has not developed with the theory of diatomic molecules based upon solutions of the one-electrontwo-nucleus problem. Without doubt, the complexity of these functions has been a deterrent to such investigations, but the present development of computing machinery makes such difficult situations tractable, at least numerically.

The simplest two-center system, $\mathrm{H}_{2}{ }^{+}$, has been investigated with various numerical techniques and forms of solution; some of the results are compared by De Carlo and Griffing. ${ }^{1}$ The earlier treatments give calculations of the ground-state energy only. More recently, Bates et al..$^{2}$ obtained solutions for 10 of the lower states (eight $\sigma$ and two $\pi$ states) of $\mathrm{H}_{2}{ }^{+}$; they included tables of expansion coefficients and the energies for each of these states.

This article is a report of work done to obtain eigen-

* Based in part on a thesis submitted by L. Y. Wilson to the Graduate Faculty of the University of Nebraska, in partial fulfilment of the requirements for the Ph.D. degree.

† National Science Foundation Science Faculty Fellow, 19611962 , and National Science Foundation Nebraska Cooperative College Teacher Development Program Fellow, 1962-1963. Present address: Chemistry Department, Union College, Lincoln 6, Neb.

1 V. De Carlo and V. Griffing, I. Phys. Chem. 66, 845 (1962).

${ }^{2}$ D. R. Bates, K. Ledsham, and A. L. Stewart, Phil. Trans. Roy. Soc. (London) A246, 215 (1953). values and eigenfunctions for some of the cases with higher and differing nuclear charges.

\section{SOLUTION OF SCHRÖDINGER'S EQUATION}

An excellent review of the studies made on the one-electron-two-center problem is provided by an article by Buckingham. ${ }^{3}$ We give here only a brief outline of the development of the solutions of the present problem.

Schrödinger's equation for the motion of one electron in the field to two infinitely massive nuclei is

$$
-\frac{1}{2} \nabla^{2} \psi-\left(\frac{Z_{1}}{r_{1}}+\frac{Z_{2}}{r_{2}}-\frac{Z_{1} Z_{2}}{\rho}\right) \psi=E \psi
$$

in atomic units, where $r_{1}$ and $r_{2}$ refer to the distances between the electron and the nucleus of charge $Z_{1}$ and $Z_{2}$, respectively, and $\rho$ refers to the distance between nuclei. As is well known, the introduction of ellipsoidal coordinates allows the separation of variables in (1) and

$$
\psi(\eta, \xi, \phi)=T(\eta) S(\xi) \Phi(\phi) .
$$

The separated differential equations are ${ }^{4}$

$$
\begin{aligned}
& \frac{d}{d \eta}\left[\left(1-\eta^{2}\right) \frac{d T}{d \eta}\right]-\left(\epsilon \eta^{2}+\rho \Delta \eta+\frac{m^{2}}{1-\eta^{2}}\right) T=-\lambda T, \\
& \frac{d}{d \xi}\left[\left(\xi^{2}-1\right) \frac{d S}{d \xi}\right]+\left(\epsilon \xi^{2}+\rho \Sigma \xi-\frac{m^{2}}{\xi^{2}-1}\right) S=\lambda S,
\end{aligned}
$$

${ }^{3}$ R. A. Buckingham, Quantum Theory, D. R. Bates, Ed. (Academic Press Inc, New York, 1961), Vol. 1.

${ }^{4}$ W. G. Baber and H. R. Hasse, Proc. Cambridge Phil. Soc. 31, 564 (1935). 
and

where

$$
d^{2} \Phi / d \phi^{2}=-m^{2} \Phi
$$

$$
\begin{aligned}
\epsilon & =p^{2} E_{e} / 2, \\
E_{\mathrm{s}} & =E-\left(Z_{1} Z_{2} / \rho\right), \\
\Delta & =Z_{1}-Z_{2}, \quad\left(Z_{1} \geq Z_{2}\right),
\end{aligned}
$$

and

$$
\Sigma=Z_{1}+Z_{2} \text {. }
$$

In this study we have used

$$
S(\xi)=\left(\xi^{2}-1\right)^{|m| / 2} \exp (-\alpha \xi)\left(\frac{2}{\xi+1}\right)^{s} \sum_{k=0}^{\infty} b_{k}\left(\frac{\xi-1}{\xi+1}\right)^{k}
$$

and

$$
T(\eta)=\sum_{l=0}^{\infty} a_{l} \Theta_{l^{m}}(\eta)
$$

where the $\theta_{l}^{m}(\eta)$ are normalized associated Legendre orthogonal functions. The expression (6) is due to Jaffe..$^{5}$ Introduction of (6) and (7) into (3) and (4) leads to two recursion relations, one for the coefficients $a_{l}$ and one for the coefficients $b_{k}$. These may be treated as matrix eigenvalue problems and simultaneous solu-

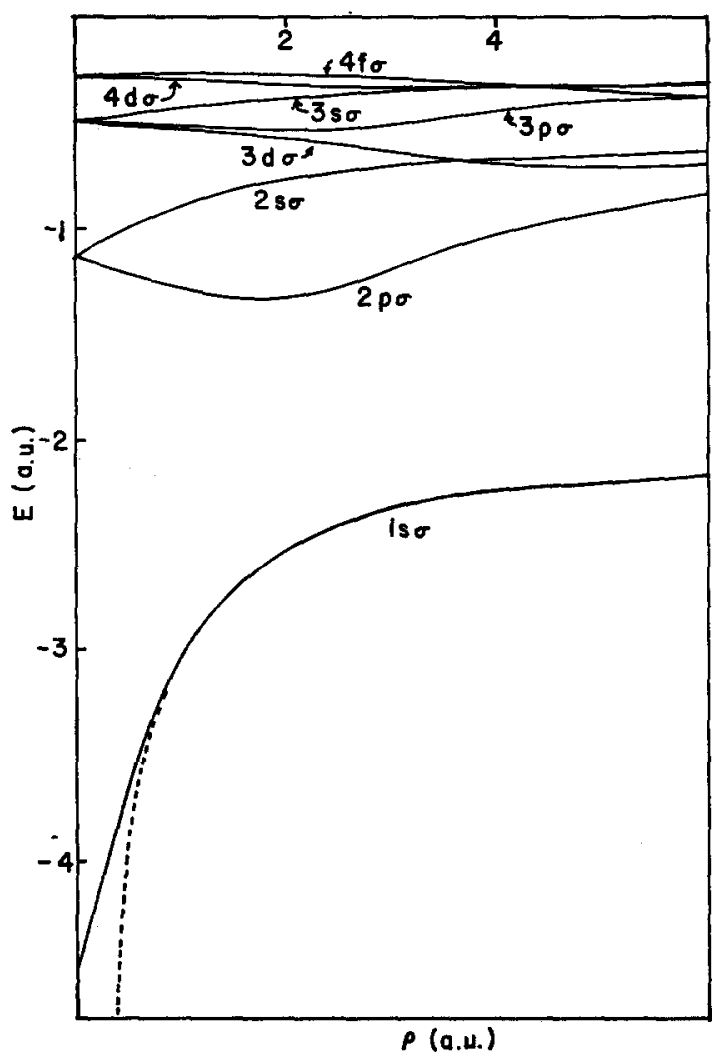

Fig. 1. First eight $\sigma$ state electronic energies as functions of the internuclear distance for $\mathrm{HeH}^{2+}$. The dashed line is calculated with (8).

B G. Jaffe, Fortschr. Physik 87, 535 (1934).

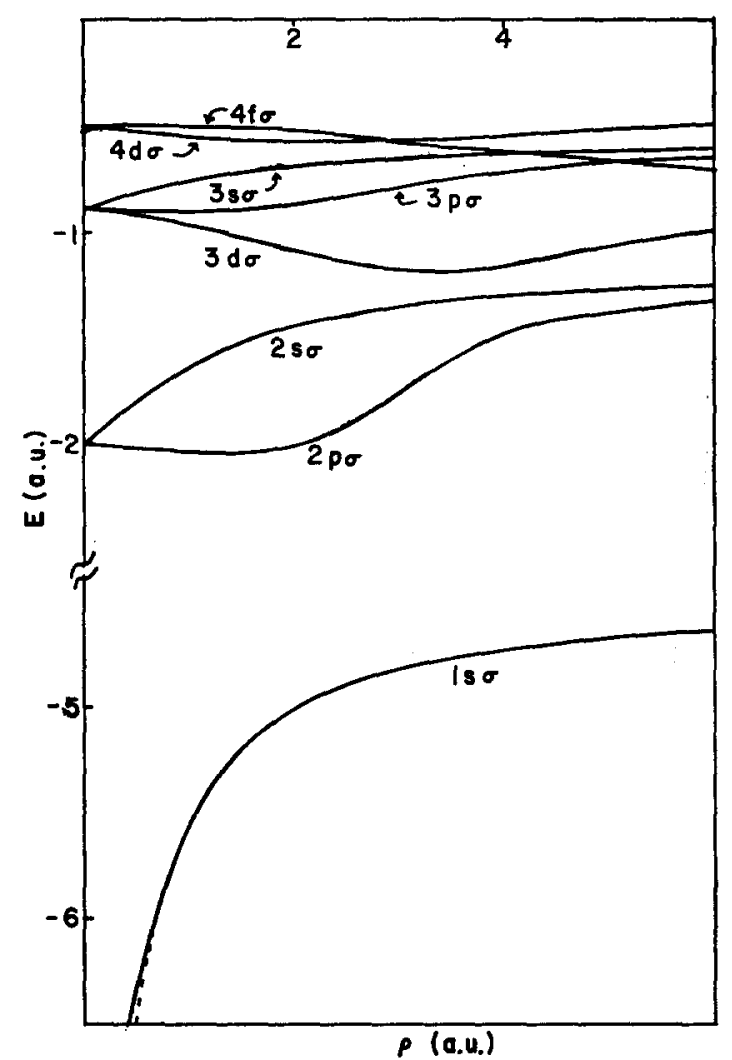

FIG. 2. First eight $\sigma$ state electronic energies as functions of the internuclear distance for $\mathrm{LiH}^{3+}$. The dashed line is calculated with (8).

tion for $\epsilon$ and $\lambda$ leads to the eigenvalues of (1). The states of the system may be given designations which correlate with the limiting case of the united atom. The present article follows the system given originally by Bates $e t a l .^{2}$

Calculations of $E_{e}(\rho)$ have been carried out for the systems $\mathrm{HeH}^{2+}, \mathrm{He}_{2}{ }^{3+}$, and $\mathrm{LiHe}^{4+}$, and the curves for the first eight $\sigma$ states of each case is shown in Figs. 1 through 4 . We have plotted $E_{e}$ rather than the total energy, since addition of the term $Z_{1} Z_{2} / \rho$ obscures both the ordering and the limiting values of the levels for small $\rho$ values.

Tables I, II, and III give our results for $\epsilon, \lambda, a_{l}$, and $b_{k}$ for $\mathrm{HeH}^{+}$at the respective $\rho$ values of $2.00,4.00$, and 6.00 a.u. Numerical results for the other cases can be obtained through University Microfilm Service. ${ }^{6}$

Bates and Carson ${ }^{7}$ suggest the inclusion of the factor $\exp \left[-(-\epsilon)^{3}\right]$ in the function $T(\eta)$ for the heteronuclear cases, which transforms the recursion relation for the coefficients $a_{l}$ into one involving only three terms. Table IV compares our results with those of Bates and Carlson for the $1 s \sigma$ state of $\mathrm{HeH}^{2+}$ at $\rho=2.00$ a.u.

${ }^{6}$ L. Y. Wilson, Ph.D. thesis, University of Nebraska, 1964.

7 D. R. Bates and T. R. Carlson, Proc. Roy. Soc. (London) A234, 207 (1956). 


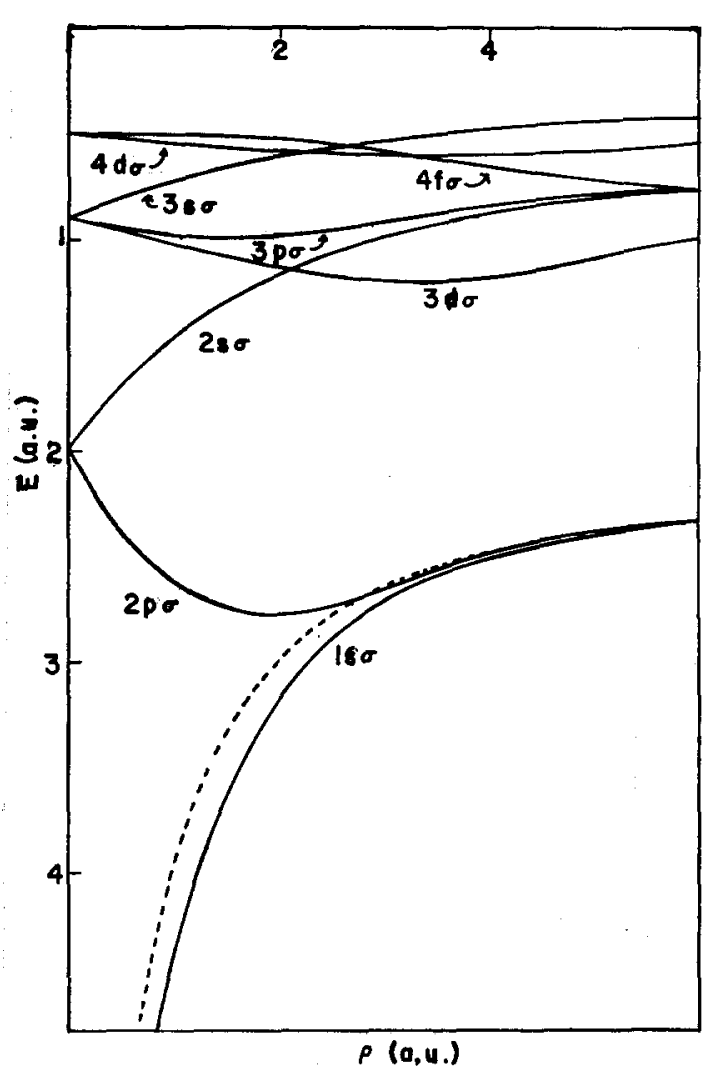

FIG. 3. First eight $\sigma$ state electronic energies as functions of the internuclear distance for $\mathrm{He}_{2}{ }^{3+}$. The dashed line is calculated with (8).

As can be seen the rate of convergence is very close to the same in the two cases at this internuclear distance. For large $\rho$ values the exponential factor should improve the convergence rate significantly.

\section{DISCUSSION}

Examination of (3) and (4) shows that $\epsilon$ and $\lambda$ depend only upon $\rho \Delta$ and $\rho \Sigma$ not upon $\rho, \Delta$, and $\Sigma$ separately. Introduction of the variables $\rho^{\prime}=\rho \Sigma$ and $\zeta=\Delta / \Sigma$ allows one to write $\epsilon_{i}=\epsilon_{i}\left(\rho^{\prime}, \zeta\right)$ and $\lambda_{i}=$ $\lambda_{i}\left(\rho^{\prime}, \zeta\right)$. Hence $E_{i} / \Sigma^{2}=\left(2 / \rho^{\prime 2}\right) e_{i}$ and is a function of $\rho^{\prime}$ and $\zeta$ only. This fact allows one to scale the results from one set of $\rho, Z_{1}$, and $Z_{2}$ to another. The scaling for the special case $\Delta=0$ was originally pointed out by Bates $e t a l .^{2}$

Baber and Hasse ${ }^{4}$ derived an asymptotic expression for the total ground-state energy of a one-electrontwo-nucleus system. If their formula is rewritten for the electronic energy only, one has

$$
E_{e}=-\left(Z_{1}^{2} / 2\right)-\left(Z_{2} / \rho\right) ; \quad Z_{1} \geq Z_{2}, Z_{1} \neq 1
$$

The physical interpretations of (8) may be clarified by use of the virial theorem, ${ }^{8}$

$$
2 \bar{T}_{e}=-\bar{V}_{e}-\rho\left(d E_{e} / d \rho\right) .
$$

One easily obtains

$$
\bar{T}_{e}=\frac{1}{2} Z_{1}^{2},
$$

and

$$
\bar{V}_{e}=-Z_{1}^{2}-\left(Z^{2} / \rho\right) .
$$

It is evident that (8) represents the energy of a normal hydrogenlike atom of nuclear charge $Z_{1}$, plus the Coulombic energy of the electron with a nucleus of charge $Z_{2}$ at an average reciprocal distance of $1 / \rho$. The original derivation indicates that (8) holds for large values of $\rho$. The dashed lines in Figs. 1 through 4 show (8) plotted for the respective nuclear charges. It is of interest that the deviations of (8) from the exact energy are less than $0.5 \%$ for $\rho=2$ for each of the heteronuclear cases. This indicates that the nucleus of smaller charge has very little influence on the shapes

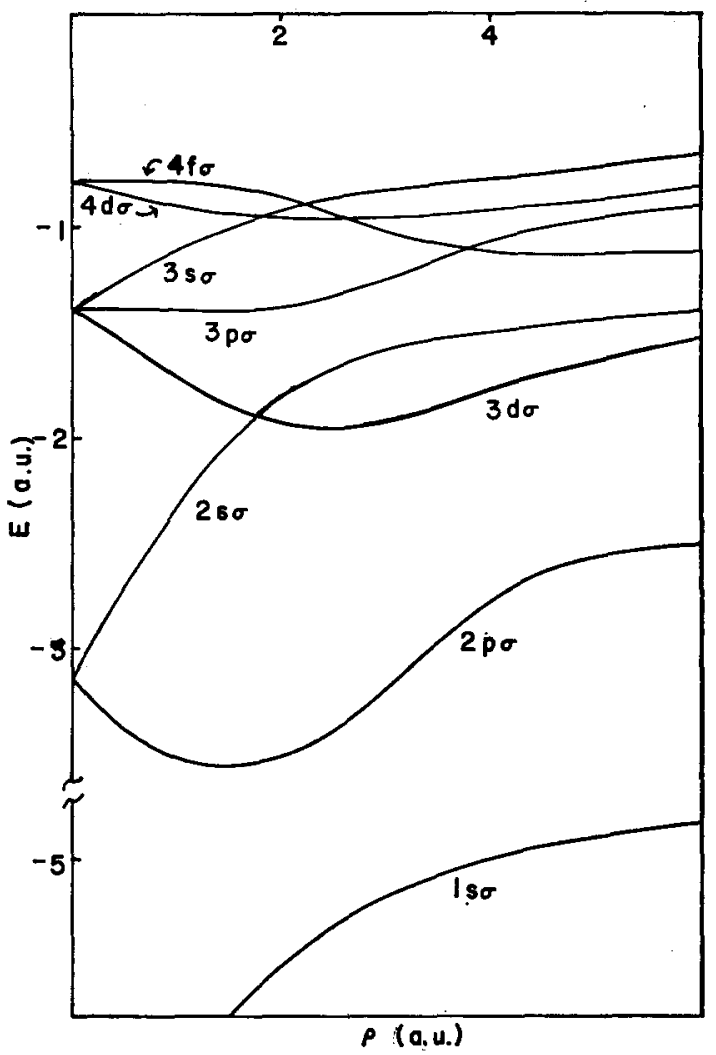

FIG. 4. First eight $\sigma$ state electronic energies as functions of the internuclear distance for $\mathrm{LiHe}^{4+}$. Results calculated with (8) are indistinguishable from the $1 s \sigma$ values on this graph.

${ }^{8}$ See, e.g., J. C. Slater, Quantum Theory of Molecules and Solids (McGraw-Hill Book Co., Inc., New York, 1963). 
ONE-ELECTRON-TWO-CENTER PROBLEM

TABLE I. Calculated values for $\mathrm{HeH}^{2+}$ at $\rho=2.00$ a.u.

\begin{tabular}{|c|c|c|c|c|c|c|c|c|c|}
\hline & & $1 s \sigma$ & $2 p \sigma$ & $2 s \sigma$ & $3 d \sigma$ & $3 p \sigma$ & $3 s \sigma$ & $4 d \sigma$ & $4 f_{\sigma}$ \\
\hline & $\begin{array}{l}\epsilon \\
\lambda\end{array}$ & $\begin{array}{l}-5.02435 \\
-3.15781\end{array}$ & $\begin{array}{r}-2.69037 \\
0.95642\end{array}$ & $\begin{array}{l}-1.57419 \\
-1.27218\end{array}$ & $\begin{array}{r}-1.14252 \\
5.49329\end{array}$ & $\begin{array}{r}-1.07325 \\
1.75924\end{array}$ & $\begin{array}{l}-0.077761 \\
-0.89809\end{array}$ & $\begin{array}{r}-0.62083 \\
5.76684\end{array}$ & $\begin{array}{c}-0.57805 \\
11.7491\end{array}$ \\
\hline$a_{l}$ & $\begin{array}{r}1=0 \\
1 \\
2 \\
3 \\
4 \\
5 \\
5 \\
6 \\
7\end{array}$ & $\begin{array}{r}-0.7482 \\
0.5930 \\
-0.2835 \\
0.0870 \\
-0.0222 \\
0.0048 \\
-0.0008 \\
0.0001\end{array}$ & $\begin{array}{r}0.5626 \\
0.8131 \\
-0.1293 \\
0.0734 \\
-0.0094 \\
0.0022 \\
-0.0002 \\
\ldots\end{array}$ & $\begin{array}{r}0.8562 \\
-0.4945 \\
0.1463 \\
-0.0286 \\
0.0043 \\
-0.0005 \\
\ldots \\
\ldots\end{array}$ & $\begin{array}{c}-0.00729 \\
0.2456 \\
0.9561 \\
-0.1569 \\
0.0316 \\
-0.0032 \\
0.0003 \\
\ldots\end{array}$ & $\begin{array}{r}0.4919 \\
0.8442 \\
-0.2076 \\
0.0468 \\
-0.0059 \\
0.0007 \\
\ldots \\
\ldots\end{array}$ & $\begin{array}{r}0.8788 \\
-0.4645 \\
0.1079 \\
-0.0165 \\
0.0019 \\
-0.0002 \\
\ldots \\
\ldots\end{array}$ & $\begin{array}{r}0.0187 \\
0.2497 \\
0.9544 \\
-0.1607 \\
0.0227 \\
-0.0020 \\
0.9002 \\
\ldots\end{array}$ & $\begin{array}{r}-0.0022 \\
0.0022 \\
0.1673 \\
0.9781 \\
-0.1227 \\
0.0149 \\
-0.0011 \\
0.0001\end{array}$ \\
\hline$b_{k}$ & $\begin{array}{r}k=0 \\
1 \\
2 \\
3 \\
4\end{array}$ & $\begin{array}{c}0.9999 \\
0.0112 \\
0.0002 \\
\ldots \\
\ldots\end{array}$ & $\begin{array}{l}0.9952 \\
0.0978 \\
0.0002 \\
\ldots \\
\ldots\end{array}$ & $\begin{array}{r}-0.2133 \\
0.9768 \\
0.0194 \\
0.0004 \\
\ldots .\end{array}$ & $\begin{array}{c}0.7182 \\
0.6944 \\
0.0440 \\
0.0001 \\
\ldots .\end{array}$ & $\begin{array}{r}-0.3143 \\
0.9401 \\
2.1323 \\
0.0001 \\
\ldots\end{array}$ & $\begin{array}{r}0.0900 \\
-0.6085 \\
0.7884 \\
0.1794 \\
0.0004\end{array}$ & $\begin{array}{r}-0.4664 \\
0.3936 \\
0.7964 \\
0.0529 \\
0.0001\end{array}$ & $\begin{array}{l}0.1744 \\
0.8550 \\
0.4875 \\
0.0304 \\
0.0001\end{array}$ \\
\hline
\end{tabular}

TABLE II. Calculated values for $\mathrm{HeH}^{2+}$ at $\rho=4.00$ a.u.

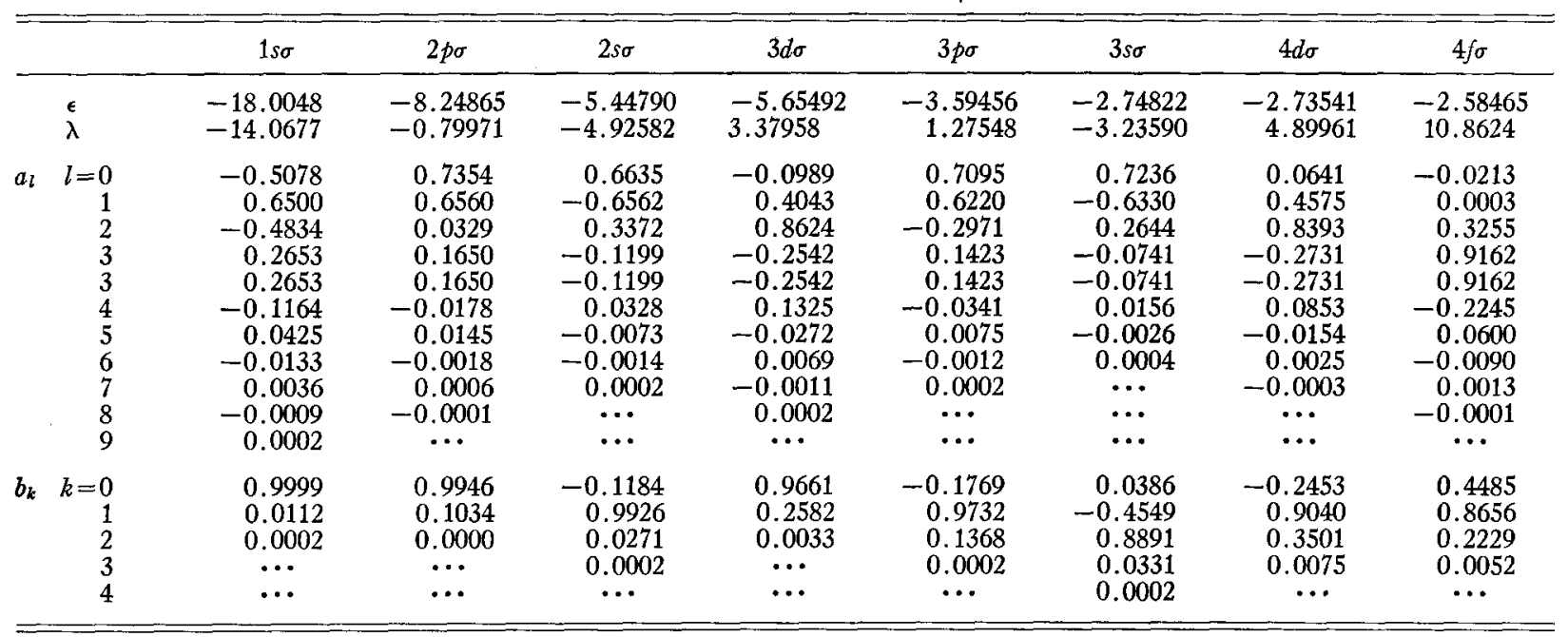

TABLE III. Calculated values for $\mathrm{HeH}^{2+}$ at $\rho=6.00$ a.u.

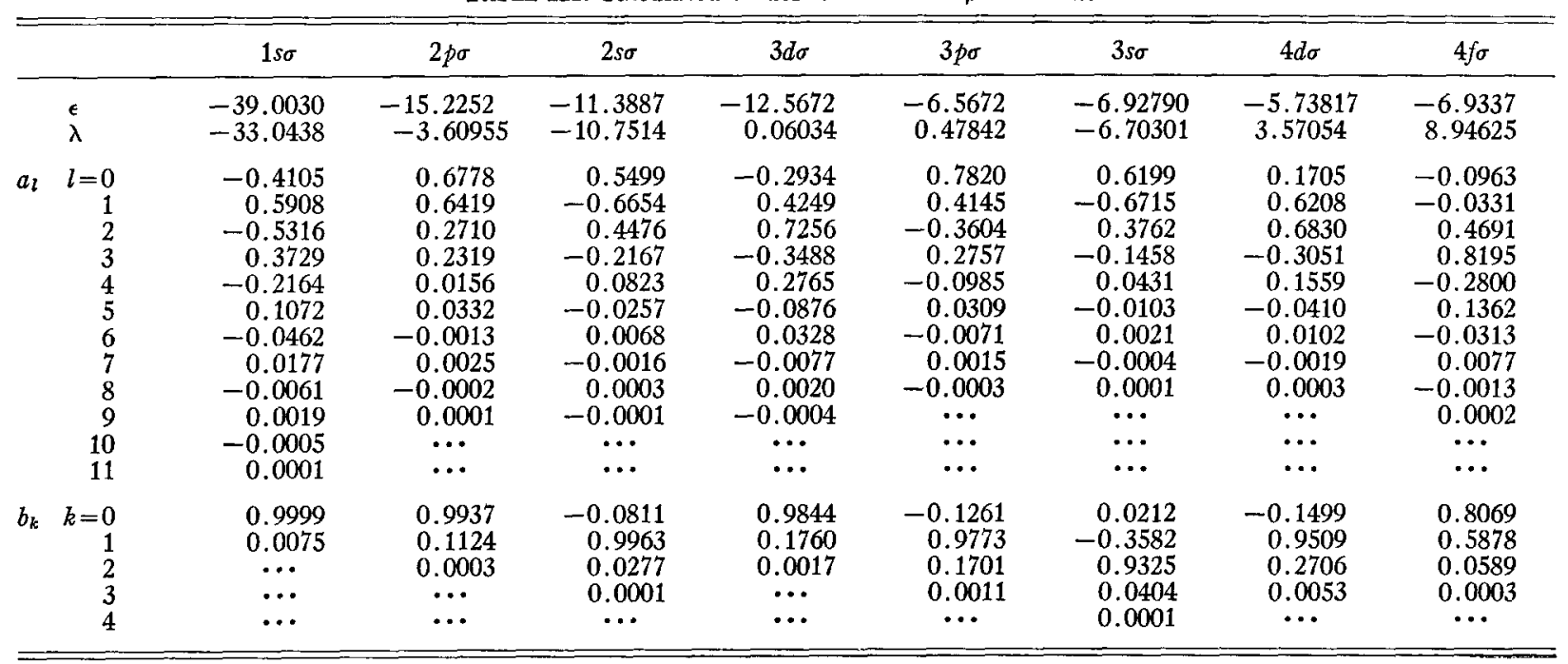




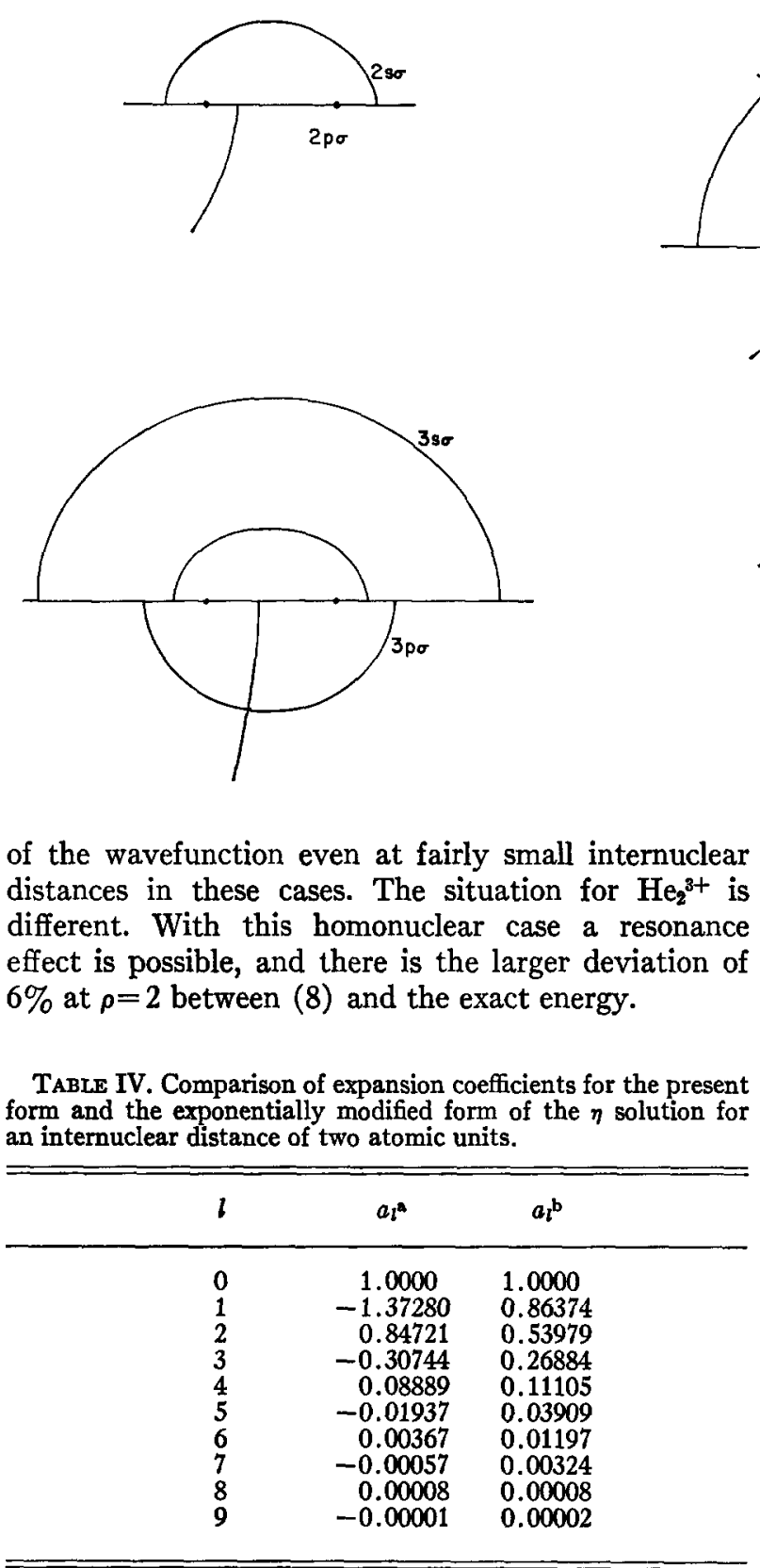

a From solution of (7). These have been modified to the coefficients for unnormalized associated Legendre functions for direct comparison.

b From Bates and Carson.?
FIG. 5. The nodal surfaces for the first eight $\sigma$ states of $\mathrm{HeH}^{2+}$ at $\rho=2.00$ a.u.

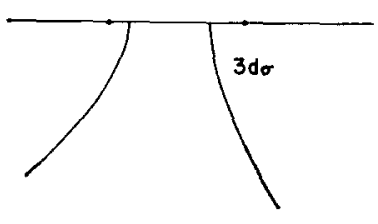

In spite of the small effect on the average kinetic energy of the electron the presence of the second nucleus has a profound effect on the shapes of the nodal surfaces of $\psi$. This follows immediately from the form of $\psi$. Restricting the discussion to $\sigma$ states, it is seen that the nodal surfaces are coordinate surfaces of the ellipsoidal system determined by $S(\xi)=0$ and $T(\eta)=0$. Hence the nodal surfaces are ellipsoids of revolution and one sheet of hyperboloids of revolution of two sheets. Figure 5 shows the intersections of the nodal surfaces with the $x-z$ plane for the $2 s \sigma, 2 p \sigma, 3 s \sigma, 3 p \sigma$, $3 d \sigma, 4 d \sigma$, and $4 f \sigma$ states of $\mathrm{HeH}^{2+}$ at $\rho=2.00$ a.u. It is interesting that, in spite of what might be expected, the ellipsoidal nodes are symmetric with respect to reflections $z \rightarrow-z$ ( $z$ axis along the internuclear line) even for the heteronuclear cases and regardless of the value of $\rho$. The positions of the hyperboloidal nodes depend strongly on $\Delta$, of course.

Examination of Table I shows that $\lambda$ approaches the eigenvalues of the total angular momentum operator as the electronic energy increases. Similar behavior is exhibited by the other systems as may be seen from Ref. 6. 